\title{
The Near-Death Experience: A Theoretical Summarization
}

\author{
Craig R. Lundahl, Ph.D. \\ Western New Mexico University
}

\begin{abstract}
This article (a) outlines a systematic theory of the near-death experience (NDE) based on accumulated research; (b) formulates 23 propositions that describe the NDE developmental process and illustrates these propositions and their relationship to one another; and (c) discusses observations on the sequence of events for the NDE and its aftermath and illustrates a proposed model of causal relationships for the NDE.
\end{abstract}

Since 1975 scholars in the field of near-death studies have produced research studies and grand theories to explain the near-death experience (NDE). More explanations have been proposed than have been adequately tested. No less than 100 publications have been concerned with explanations for the NDE and rebuttals to these explanations. Many of these theories have been quite controversial. What seems to be needed in the field of near-death studies is a comprehensive theory of the NDE. Such a theory should be based on the accumulated research in the field over the past 18 years, and could help resolve the debate over an explanation for the NDE.

Modern research on the NDE began when psychiatrist Raymond Moody collected more than 150 accounts of the NDE. He identified 15 elements that often recurred in these accounts: a sense of ineffability, pronouncements of death, feelings of peace, a buzzing or ringing noise,

Craig R. Lundahl, Ph.D., is Professor of Sociology and Business Administration and Chair of the Department of Social Sciences at Western New Mexico University. This research was supported by a sabbatical leave granted by the Sabbatical Leave Committee of Western New Mexico University. 
moving through a dark void or tunnel, separation from the physical body, meeting others, an encounter with a being of light, a panoramic life review, an approach to a border or limit, return to the physical body, telling others of the experience, lasting effects on lives, new views on death, and subsequent corroboration of the experience (Moody, 1975). He followed this study with a later publication adding the new NDE components of the vision of knowledge, cities of light, a realm of bewildered spirits, and supernatural rescues (1977).

Maurice Rawlings (1978) reported that more than 20 percent of the patients he resuscitated described NDEs. He reported a composite experience including a sense of hovering above the body, acute perceptions, moving through a tunnel, a realm of light, encountering others, a being of light, life review, judgment, and a boundary. He also reported some hellish NDEs.

Michael Sabom and Sarah Kreutziger (1978) found 61 NDEs in 100 patients who had been unconscious and near death. Sixteen patients viewed their bodies from a detached position, which they called autoscopy; 31 experienced passage of consciousness into a foreign dimension, which they called transcendence; and 13 experienced both autoscopy and transcendence. I examined 11 accounts of Mormon NDEs, nine of them occurring before 1923 (Lundahl, 1979, 1982). All the subjects experienced Moody's common elements except ineffability, the noise, the border or limit, and vision of knowledge.

Kenneth Ring $(1979,1980,1984)$ surveyed 102 near-death survivors, half of whom reported experiences containing NDE elements. Of those who had NDEs, 60 percent experienced peace, 37 percent experienced separation from the body, 23 percent experienced entering the darkness, 16 percent experienced seeing the light, and 10 percent experienced entering the light. These NDErs often reported a decision point of return that tended to follow a life review and an encounter with a presence or meeting with loved ones. Ring found that the incidence and depth of the experiences were greatest for illness victims, moderate for accident victims, and lowest for suicide attempters. He found no correlations between the likelihood and depth of NDEs and various demographic measures, and his data largely confirmed Moody's findings.

James Lindley, Sethyn Bryan, and Bob Conley (1981) analyzed 49 NDErs' accounts and reported that 75 percent reported a sense of peace; 71 percent, an out-of-body experience; 38 percent, a tunnel or void; 56 percent, the light; and 35 percent, a paradisical setting. Timothy Green and Penelope Friedman (1981) reported a study of 50 NDEs in which 70 percent described a sense of peace; 66 percent, an out-of- 
body experience; 32 percent, a tunnel or dark area; 62 percent, seeing the light; and 18 percent, entering the light.

Pollster George Gallup (Gallup and Proctor, 1982) found in a national survey of NDEs that 11 percent of NDErs reported experiencing a sense of peace; 11 percent, a life review; 11 percent, a sense of being in a different world; 9 percent, an out-of-body sensation; 8 percent, acute visual perception of surroundings; 8 percent, the presence of a special being or beings; 6 percent, audible sounds of human voices; 5 percent, the light; 3 percent, a tunnel; 2 percent, premonitions; and 1 percent, a sense of hell or torment.

Sabom (1982) determined through an analysis of 61 nonsurgical NDE cases that the NDE content consisted of 10 elements: a sense of being dead ( 92 percent), a sense of peace (100 percent), separation from the body ( 100 percent), autoscopy ( 53 percent), a dark region or void ( 23 percent), a life review (3 percent), entering a transcendent realm (54 percent), encountering others ( 48 percent), the light ( 28 percent), and the return to the body (100 percent).

Ring and Stephen Franklin (1981-82) surveyed 17 suicide survivors, who reported they experienced all of the various elements commonly found in accounts of NDEs. Paola Giovetti (1982) studied 120 Italian accounts of NDEs and other deathbed phenomena, and found 46 included appearances of deceased relatives or friends; 29 , out-of-body experiences; 21 , heavenly landscapes or realms; 17 , the perception of a barrier or limit; and 11, phenomena such as lights, raps, voices, and apparitions.

Margot Grey (1985) assessed the NDE reports of 32 English and nine American subjects and found that 38 of the respondents described events that fell within one or more categories of Ring's core experience model. Most of her subjects reported a sense of joy and separation from the body, while decreasing numbers described the deeper stages of entering the darkness, seeing the light, and experiencing another world. An eighth of her sample reported hellish experiences.

Nancy Bush (1983) reported that the experiences most often found in 17 accounts of NDEs of children were the light, a sense of well-being, separation from the body, entering the darkness or tunnel, peace, absence of fear, and spiritual presences. She reported that except for the lack of a life review and any sense of being judged, the features of children's NDEs were comparable in content to those reported by adults.

Melvin Morse, Paul Castillo, David Venecia, Jerrold Milstein, and Donald Tyler (1986) reported on seven childhood NDEs. Six of these children experienced being out of the body; 5 , entering the darkness; 
4 , being in a tunnel; and 3, deciding to return. William Serdahely (1990) reported research suggesting that childhood NDEs include feeling pain-free, seeing a light at the tunnel's end, entering the light, and time alteration. His cases also suggested that the life review may be a function of chronological age.

This review indicates that there is now enough research in the field of near-death studies on which to build a systematic theory of the NDE. Such a theory should address who has an NDE and why, what is the content of the NDE, and what influences the NDE. This article is a step toward development of such a systematic theory of the NDE based on accumulated research. This theory is intended to deal with the NDE as a process rather than as a series of independent and measurable variables.

\section{Propositions for a Theory of Near-Death Experiences}

A theory is used to systematize and organize experiences like the NDE. A good theory should organize what we know from prior research as well as generate propositions that can be tested in future research. Any theory consists of a set of propositions that are developed to describe a relationship between variables and explain a certain phenomenon. Below are developed a number of propositions that describe the variables of the NDE and the NDE process itself.

The first proposition that might be considered the necessary condition for, or barrier to beginning, the NDE process:

Proposition 1: The likelihood that an individual will come close to death is increased by a serious illness, serious accident, or suicide attempt.

The three major modes of near-death onset in Proposition 1, a serious illness, a serious accident, or a suicide attempt, may bring people close to death. Although some researchers, such as Moody (1977) and Gallup (Gallup and Proctor, 1982), have suggested several possible modes of near-death onset, those modes that are appropriate in light of research have been subsumed under the abovementioned three major modes.

The second proposition might be considered a necessary condition for an NDE:

Proposition 2: A closeness to death makes possible, and is a precondition for, a near-death experience. 
This proposition is based on Morse's work (Morse and Perry, 1990) showing that a person actually needs to be near death to have an NDE. Reports of NDEs by other researchers have generally been of individuals who were near death. However, some studies of adult NDErs have suggested that NDEs may be as common among people who think they are near death as they are among people who actually are near death (Greyson, 1989). Further research may offer firmer evidence for this observation. Research also shows that demographic variables do not influence the likelihood of having an NDE or, for that matter, the depth of an NDE. In other words, closeness to death is a precondition to having an NDE.

Another factor that has been proposed as an early perpetuator of the NDE is unconsciousness (Sabom, 1982):

Proposition 3: The likelihood of an NDE increases as unconsciousness is prolonged.

Duration of unconsciousness affects the incidence of NDEs: the NDE may either be perpetuated or reduced by prolonged or shortened unconsciousness.

NDE incidence is greatest for illness victims, moderate for accident victims, and lowest for those who attempt suicide (Ring, 1980). Thus, keeping in mind that the discussion so far is only on the period before the NDE begins:

Proposition 4: The incidence of an NDE is greatest for illness victims, moderate for accident victims, and less for suicide attempters.

If a person is close to death, it is possible he or she may have an NDE. According to Gallup's national survey (Gallup and Proctor, 1982), about 35 percent of all people who have a verge-of-death or temporary death experience have an NDE.

The NDE usually begins with a feeling of peace and/or well-being as well as with a loss of pain. An experience of peace was described by 100 percent of Sabom's subjects (1982), 74 percent of the subjects in Lindley, Bryan, and Conley's study (1981), 70 percent in Green and Friedman's study (1983), 60 percent of Ring's subjects $(1979,1980)$, and 11 percent of the Gallup Poll national sample (Gallup and Proctor, 1982). These findings are reflected in Proposition 5:

Proposition 5: The likelihood that the individual close to death will experience a feeling of peace and well-being and of painlessness is increased by having an NDE. 
Once the NDE has begun, a barrier to communication may be confronted. Efforts to resuscitate the NDEr may terminate the NDE. It is probably because of current medical technology allowing quick resuscitation of patients that many NDErs do not proceed to deeper levels of the experience. Proposition 6 addresses resuscitation efforts and the development of the NDE:

Proposition 6: The likelihood that an NDE will be terminated before reaching deeper experience stages increases with efforts to resuscitate the NDEr.

As the NDE continues, research generally suggests the next stage is a separation from the physical body. Bodily separation was described by 100 percent of Sabom's subjects (1982), 71 percent of the subjects in Lindley, Bryan, and Conley's study (1981), 66 percent of Green and Friedman's subjects (1983), 37 percent of Ring's subjects $(1979,1980$ ), and 9 percent of the Gallup Poll national sample (Gallup and Proctor, 1982). Proposition 7 summarizes this stage of the NDE:

Proposition 7: The likelihood that an NDEr will separate from the physical body increases as the NDE progresses to a deeper experience level.

Generally, once NDErs see and recognize their physical bodies from an out-of-body vantage point, they sense being dead. However, this feeling does not necessarily have to occur after seeing one's body. Sabom (1982) reported that 92 percent of his subjects experienced a sense of being dead, and other researchers also discussed this phase of the NDE (Moody, 1988; Ring, 1980). Proposition 8 addresses this next phase of the NDE:

Proposition 8: The likelihood that an NDEr will have a sense of being dead increases as the NDE progresses to a deeper experience level.

Prolonged unconsciousness perpetuates the NDE and facilitates its progression to deeper levels. The NDE is also progressed to deeper experience levels by occurrence of an apparent clinical death and by the prolongation of that clinical death, observations made by Moody (1975). The following propositions describe the perpetuation of an NDE and its progression to deeper levels:

Proposition 9: The likelihood that an NDE will progress beyond a minimal experience increases as unconsciousness is prolonged. 
Proposition 10: The likelihood that an NDE will progress to deeper experience levels is increased by the NDEr undergoing an apparent clinical death.

Proposition 11: The likelihood that an NDE will progress to deeper experience levels is increased with the duration of apparent clinical death.

Research findings suggest that as NDErs move into a moderately deep experience, they may enter a darkness, often described as a dark tunnel or void. Entering such a dark space was described by 38 percent of subjects in Lindley, Bryan, and Conley's study (1981), 32 percent Green and Friedman's subjects (1983), 23 percent of Ring's subjects $(1979,1980), 23$ percent of Sabom's subjects (1982), and 3 percent of the Gallup Poll national sample (Gallup and Proctor, 1982). Proposition 12 summarizes this stage of the NDE:

Proposition 12: The likelihood that the NDEr will enter a darkness increases as the NDE progresses to a deeper experience level.

This stage of the NDE is followed by seeing a light. Sixty-two percent of Green and Freidman's subjects (1983) described seeing the light, as did 56 percent of the subjects in Lindley, Bryan, and Conley's study (1981), 28 percent of Sabom's subjects (1982), 16 percent of Ring's subjects $(1979,1980)$, and 5 percent of the Gallup Poll national sample (Gallup and Proctor, 1982). Proposition 13 summarizes this stage of the NDE:

Proposition 13: The likelihood that the NDEr will see the light increases as the NDE progresses to a deeper experience level.

As noted above, Ring (1980) found in his research that the depth of an NDE was greatest for illness victims, moderate for accident victims, and lowest for suicide attempters. Ring also reported that suicide-related NDEs in his study never reached the stages of seeing the light and entering the light. Proposition 14 summarizes this finding:

Proposition 14: The likelihood of having an experience beyond a minimal NDE is reduced by attempting suicide.

As the NDE progresses to a deeper experience level, the NDEr is likely to enter another world and encounter a being of light, a presence, and/or other beings. Entering another realm or world was 
described by 54 percent of Sabom's subjects (1982), 39 percent of subjects in Lindley, Bryan, and Conley's study (1981), 18 percent of subjects in Green and Friedman's study (1983), 11 percent of the Gallup Poll national sample (Gallup and Proctor, 1982), and 10 percent of Ring's subjects $(1979,1980)$. Proposition 15 addresses this stage of the NDE:

Proposition 15: The likelihood that the NDEr will enter another world increases as the NDE progresses to a deeper experience level.

Once NDErs are in this new environment, they usually encounter a being of light, a presence, or other beings. Sabom (1982) found that 48 percent of his subjects reported encountering others, and the Gallup Poll found that 8 percent of a national sample reported the presence of a special being or beings (Gallup and Proctor, 1982). If the NDEr encounters a presence or being of light, he or she will probably experience a life review. In the Gallup Poll, 11 percent of a national sample experienced a life review (Gallup and Proctor, 1982), as did 3 percent of Sabom's subjects (1982). Propositions 16 and 17 summarize this phase of the NDE:

Proposition 16: The likelihood that the NDEr will encounter a being of light, a presence, and/or other beings increases as the NDE progresses to a deeper experience level.

Proposition 17: The likelihood of experiencing a life review increases if an NDEr encounters a being of light or a presence.

At any time during the NDE, the experience may be terminated because of an alternative to death, that is, an opportunity to return to life. This often happens when the NDEr is asked if he or she would like to return to life following the life review, but may actually occur at any time during an NDE. Proposition 18 summarizes this barrier to continuation of the NDE:

Proposition 18: An alternative to death may arise at any phase of the NDE. The likelihood that the NDE will be terminated increases with the attractiveness to the NDEr of the alternative to death.

As Ring (1980) found, if the NDEr undergoes a serious accident, he or she is less likely to experience a deep NDE than an NDEr who undergoes a serious illness. This finding is reflected in Proposition 19:

Proposition 19: The likelihood that an individual will experience a deep NDE is reduced by having a serious accident. 
Furthermore, the more an NDE progresses to a deeper experience level, the more likely it will be terminated. This obvious observation is supported by research reports, and is reflected in Proposition 20:

Proposition 20: The likelihood that an NDE will be terminated increases as the NDE progresses to a deeper experience level.

Once NDErs have encountered a presence, a being of light, or other beings, or undergone a life review, they are often asked to decide whether to return to life, or are told to return to life. When NDErs are given the option of returning to life, they usually decide to return to the body, or if reluctant to do so, are soon persuaded to return. It is a certainty that when NDErs are told to return to life, they do so. This brings us to the final three propositions:

Proposition 21: The likelihood of being asked to decide whether to return to life or of being told to return to life increases if an NDEr encounters a being of light, a presence, or other beings, or experiences a life review.

Proposition 22: The likelihood of returning to the body increases if the NDEr is asked to decide whether to return to life.

Proposition 23: Return to the body is a certainty if the NDEr is told to return to life.

These 23 propositions constitute the basis for a propositional and systematic theory of the NDE based on the accumulated research in the field of near-death studies. These various propositions are incorporated into a developmental process of the NDE shown in Figure 1.

\section{A General Story of the Near-Death Experience}

A good theory tells a general story of humanity and, therefore, a theory of the NDE should closely parallel the way people actually experience an NDE. To provide a better understanding of the 23 propositions of this NDE theory, let us consider them in light of a brief story of a hypothetical NDE:

Mary is seriously ill from kidney failure and multiple associated complications (Proposition 1). She is dying from her illness (Proposition 2) and as she reaches the greatest distress she loses consciousness (Propositions 3 and 4).

She begins to feel a sense of peace and well-being, and notices that she no longer feels any pain (Proposition 5). Suddenly, she finds herself separated from her body and in the air above it (Proposition 7), looking down at her physician and two nurses working to resuscitate 

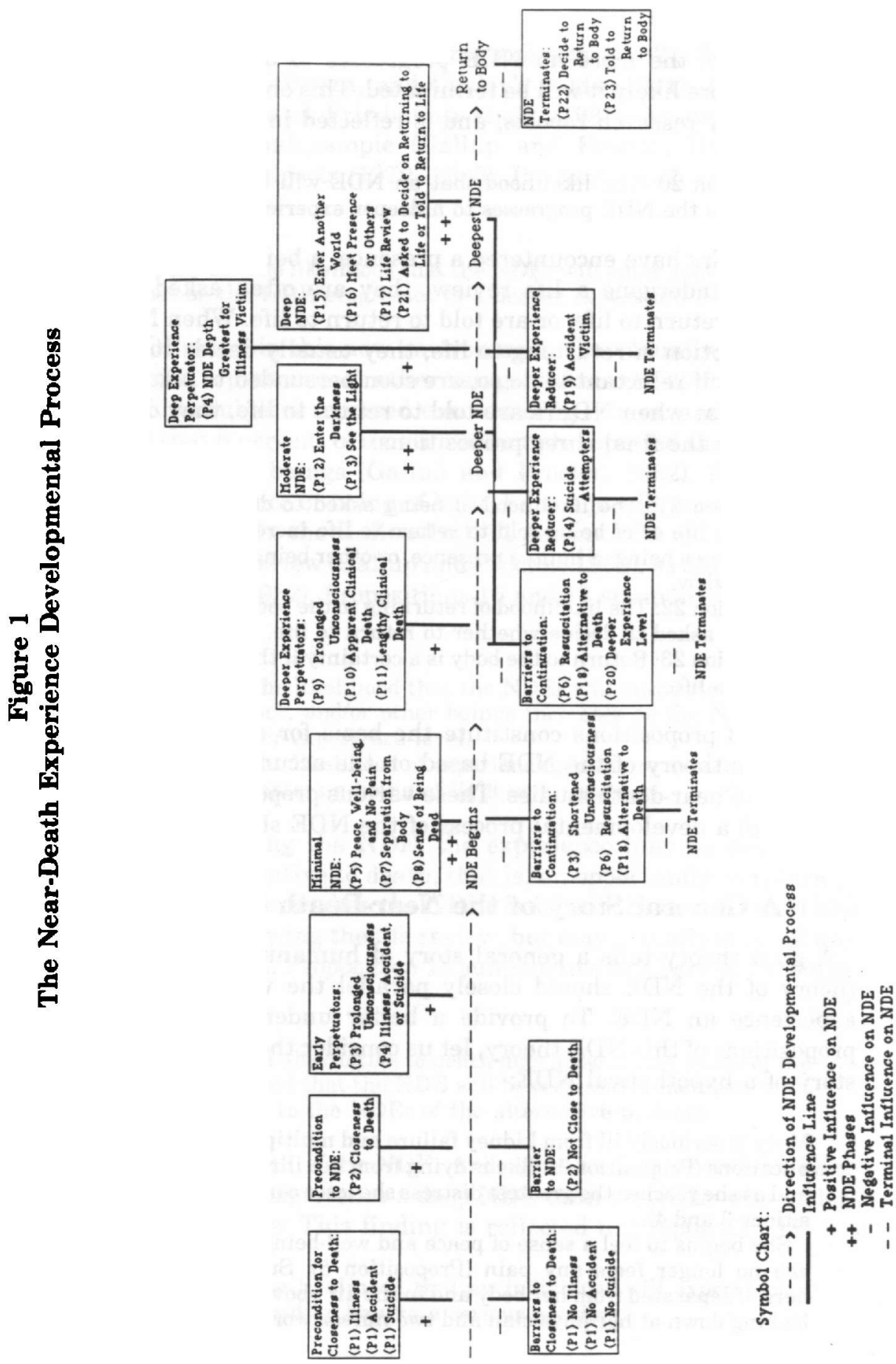
her (Proposition 6). At this point, she begins to realize she is dead but she still has a body, acute vision and hearing, and a clear and alert mental state (Proposition 8).

After 10 minutes she still remains unconscious (Proposition 9) and she is pronounced dead (Propositions 10 and 11). She then enters a dark space (Proposition 12), at the end of which she sees a brilliant light (Proposition 13). Upon entering this light (Proposition 14), she finds herself in another world of preternatural beauty (Proposition 15).

She now meets a being of light (Proposition 16) who takes her through a life review and helps her put all the events of her life in perspective (Proposition 17). The life review is a full color, threedimensional, panoramic review of every single thing Mary has done in her life, in what seems to be an instant. She perceives the effects of all her actions upon the people in her life.

At this point in her NDE, she considers an alternative to death, and thinks about what it might be like to return to her body (Proposition 18). But Mary, who has been suffering from a serious illness (Proposition 19), continues her deep NDE (Proposition 20).

Eventually, however, Mary is asked by the being of light whether she wants to return to life and she begins to contemplate her responsibilities to her family (Proposition 21). Although she is reluctant to go back, she finally decides to do so (Proposition 22). When her spirit re-enters the body, the pain and suffering return. She eventually recovers from her illness and as a result of her NDE she loses all fear of death and experiences a change in her values, attitudes, and behaviors.

This story could just as easily have been written so that Mary meets her deceased relatives in the other world (Proposition 21), who tell her that she will have to return to life because it is not her time yet, and she returns to her body (Proposition 23). Under these circumstances, Mary may not experience a life review (Proposition 17).

\section{The Sequence of Events in the Near-Death Experience}

This propositional theory of the NDE based on accumulated research suggests an association between a sequence of events prior to the NDE and following the NDE. Generally, it can be said that there is a relationship between an NDE and changes in behavior of the NDEr. The aftereffects of the NDE on the experiencer are beyond the scope of these theoretical propositions that explain the NDE developmental process, but they have been discussed extensively in the literature (Lundahl, 1993; Morse and Perry, 1992) and are important to our understanding of events following the NDE. This relationship is asymmetrical in that 
having an NDE results in a change in the experiencer's behavior, whereas a change in behavior does not result in an NDE. In other words, an NDE at one point in time seems to cause behavior changes in the experiencer, such as a change in religious behavior (Ring, 1980), at some point in time following the NDE.

This theory also suggests that a serious illness, a serious accident, or a suicide attempt is a necessary but not sufficient condition for the occurrence of an NDE. To experience an NDE an individual must be close to death, as a result of either illness, accident, or attempted suicide. However, not all persons who come close to death have an NDE. The other factors that contribute to having an NDE have not yet been ascertained. I believe, based on some scrutiny of NDE case studies, that other factors necessary for an NDE may be spiritual purposes and the inward spirituality of the NDEr. Together, these combined factors and perhaps other as yet unidentified factors may be sufficient to cause an NDE, but each alone, while necessary, is not sufficient.

This aspect, the cause of the NDE, has been the center of considerable discussion among near-death researchers. Some researchers believe the unknown factors may be neurological, pharmacological, physiological, psychological, or religious in nature. Other researchers see a neurological, pharmacological, physiological, or psychological factor as the sole cause of the NDE, suggesting that a neurological, pharmacological, physiological, or psychological factor is simultaneously both necessary and sufficient for the occurrence of an NDE.

The NDE seems to be the same journey, with different people encountering different segments or stages of a single, common path (Ring, 1980). Research seems to point to ten general steps in this path: peace, bodily separation, sense of being dead, entering the darkness, seeing the light, entering another world, meeting others, life review, deciding to or being told to return to life, and returning to the body. The incidence and depth of the NDE itself are contingent upon the cause of the close brush with death (illness, accident, or suicide attempt) and upon length of unconsciousness. Progression to deeper NDE levels is also contingent upon resuscitation efforts, apparent clinical death and its duration, an alternative to death, and the level of depth of NDE already reached.

As noted above, there is an apparent relationship between the NDE and experiencers' behavior changes. However, this apparent correlation exists because of several other variables intervening between the NDE and the behavior changes, namely, changed values, desires, motives, and thoughts. In other words, the NDE causes changes in values, which in turn cause changes in the NDErs' desires, which in turn 
cause changes in the individuals' motives, which in turn cause changes in the experiencers' thoughts, resulting finally in changes in the NDErs' actions or behaviors. Although these behavioral changes are also seen in those who approach death or who have a death experience without experiencing an NDE, the behavioral changes are not as extensive as those of NDErs (Ring, 1980). Morse's study found that changes in behavior were most profound in NDErs who had experiences of light (Morse and Perry, 1992). These research findings suggest that the intervening variables of changed values, desires, motives, and thoughts are sufficient for the occurrence of each of the subsequent variables in the chain, and that they occur every time the preceding variables occur.

Figure 2 presents a proposed model of causal relationships for the NDE that attempts to illustrate these observations on the sequence of events for the NDE.

\section{Conclusion}

This article has attempted to take a first step in a systematic theory of the NDE based on accumulated research in the field of near-death studies, and the associations between different variables in the developmental process of the NDE to the behaviors of NDErs. This exercise

Figure 2

A Proposed Model of Causal Relationships for the Near-Death Experience

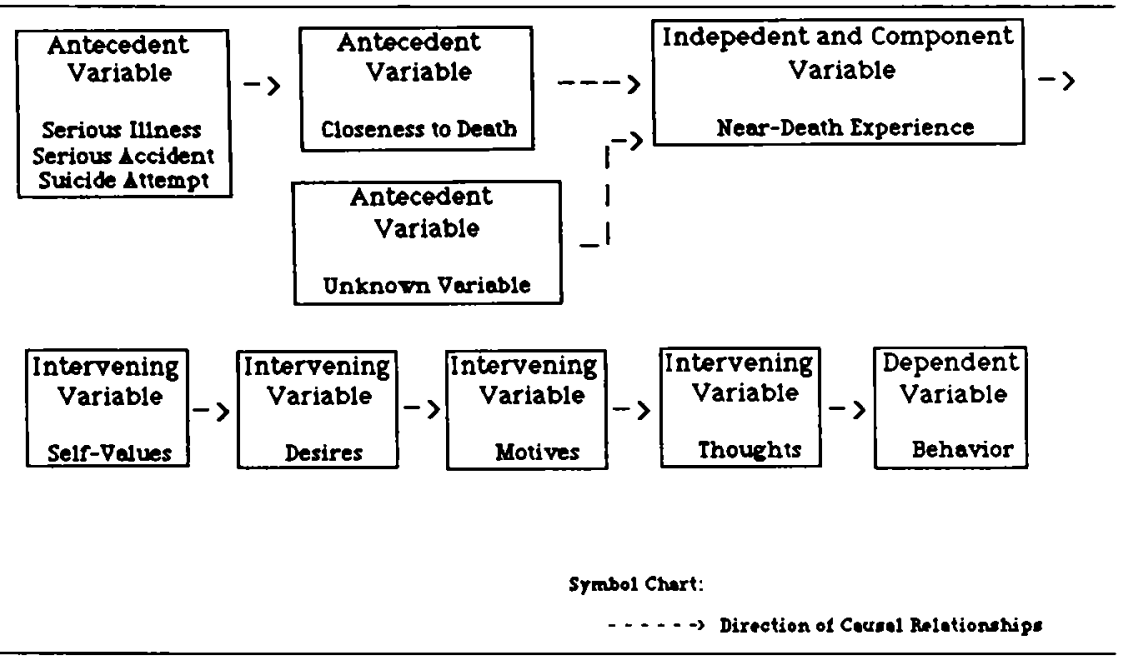


identifies the need for continuous research on identifying the factors that explain why some people experience an NDE while close to death, while others do not. Future research findings should be included in modifications to this proposed NDE theory and to the proposed causal model.

\section{References}

Bush, N.E. (1983). The near-death experience in children: Shades of the prison-house reopening. Anabiosis: The Journal of Near-Death Studies, 3, 177-193.

Gallup, G., Jr., and Proctor, W. (1982). Adventures in immortality: A look beyond the threshold of death. New York, NY: McGraw-Hill.

Giovetti, P. (1982). Near-death and deathbed experience: An Italian survey. Theta, 10(1), $10-13$.

Green, J.T., and Friedman, P. (1981). Near-death experiences in a Southern California population. Anabiosis: The Journal of Near-Death Studies, 3, 77-95.

Grey, M. (1985). Return from death: An exploration of the near-death experience. London, England: Arkana.

Greyson, B. (1989). Can we explain the near-death experience? [Editorial]. Journal of Near-Death Studies, 8, 77-92.

Lindley, J.H., Bryan, S., and Conley, B. (1981). Near-death experiences in a Pacific Northwest American population: The Evergreen Study. Anabiosis: The Journal of Near-Death Studies, 1, 104-124.

Lundahl, C.R. (1979). Mormon near-death experiences. Free Inquiry in Creative Sociology, 7, 101-104 \& 107.

Lundahl, C.R. (1982). Near-death experiences of Mormons. In C.R. Lundahl (Ed.), A collection of near-death research readings (pp. 165-179). Chicago, IL: Nelson-Hall.

Lundahl, C.R. (1993). Lessons from near-death experiences for humanity. [Guest editorial]. Journal of Near-Death Studies, 12, 5-16.

Moody, R.A., Jr. (1975). Life after life. Covington, GA: Mockingbird.

Moody, R.A., Jr. (1977). Reflections on life after life. St. Simon's Island, GA: Mockingbird.

Morse, M., Castillo, P., Venecia, D., Milstein, J., and Tyler, D.C. (1986). Childhood neardeath experiences. American Journal of Diseases of Children, 140, 1110-1114.

Morse, M., and Perry, P. (1990). Closer to the light: Learning from the near-death experiences of children. New York, NY: Villard.

Morse, M., and Perry, P. (1992). Transformed by the light: The powerful effects of neardeath experiences on people's lives. New York; NY: Villard.

Rawlings, M. (1978). Beyond death's door. Nashville, TN: Thomas Nelson.

Ring, K. (1979). Further studies of the near-death experience. Theta, 7(2), 1-3.

Ring, K. (1980). Life at death: A scientific investigation of the near-death experience New York, NY: Coward, McCann, and Geoghegan.

Ring, K. (1984). Heading toward omega: In search of the meaning of the near-death experience New York: NY: William Morrow.

Ring, K., and Franklin, S. (1981-82). Do suicide survivors report near-death experiences? Omega, 12, 191-208.

Sabom, M.B. (1982). Recollections of death: A medical investigation. New York, NY: Harper and Row.

Sabom, M.B., and Kreutziger, S. (1978). Physicians evaluate the near-death experience. Theta, 6(4), 1-6.

Serdahely, W.J. (1990). Pediatric near-death experiences. Journal of Near-Death Studies, 9, 33-39. 